\title{
Word frequency and the mixed-list paradox in immediate and delayed serial recall
}

\author{
CAROLINE MORIN \\ University of Warwick, Coventry, England \\ MARIE POIRIER \\ City University, London, England \\ CLAUDETTE FORTIN \\ Université Laval, Québec, Canada \\ and \\ CHARLES HULME \\ University of York, York, England
}

\begin{abstract}
In free recall tasks, when low- and high-frequency items are mixed within the to-be-remembered lists, the usual recall advantage found for high-frequency words is eliminated or reversed. Recently, this mixedlist paradox has also been demonstrated for short-term serial recall (Hulme, Stuart, Brown, \& Morin, 2003). Although a number of theoretical interpretations of this mixed-list paradox have been proposed, researchers have also suggested that it could simply be a result of participant-controlled strategies (M. J. Watkins, LeCompte, \& Kim, 2000). The present study was designed to assess whether this explanation could be applied to immediate and delayed serial recall. The results showed that high-frequency words were recalled better than low-frequency words in pure lists, but that this effect was eliminated in mixed lists, whether they were given under intentional or incidental learning conditions. This pattern suggests that the mixed-list paradox cannot be explained by participant-controlled strategies.
\end{abstract}

Research on memory over the short term has systematically investigated the relationship between prior representations - the knowledge that participants bring to the laboratory - and memory for the most recently presented information: the to-be-recalled list. This work has led to the identification of a series of benchmark findings that have contributed to current theoretical development. This paper is concerned with the most studied of these factors-namely, word frequency.

Until recently, it was thought that in immediate serial recall, high-frequency words were better recalled than low-frequency words (Gregg, Freedman, \& Smith, 1989; Poirier \& Saint-Aubin, 1996; Stuart \& Hulme, 2000; O. C. Watkins \& M. J. Watkins, 1977). However, Hulme, Stuart, Brown, and Morin (2003) have shown that this word frequency effect depends critically on list composition, and that the high-frequency advantage typically found in

This article was part of C.M.'s doctoral thesis. Preparation of this article was supported by a grant from the Natural Sciences and Engineering Research Council of Canada to M.P. C.M. was supported by a graduate scholarship from Laval University Foundation and then by ESRC Grant RES 000 231038. We thank Silvio Aldrovandi for running Experiment 1. We also thank Alice F. Healy, Ian Neath, and an anonymous reviewer for their comments on an earlier version of this article. Correspondence concerning this article should be addressed to C. Morin, Psychology Department, University of Warwick, Coventry CV4 7AL, England (email: caroline.morin@warwick.ac.uk). immediate serial recall is eliminated in mixed lists that contain both high- and low-frequency words.

This mixed-list paradox poses some significant challenges for current models of short-term memory. Although many models can account for the word frequency effect in pure lists (e.g., Burgess \& Hitch, 1999; Lewandowsky \& Murdock, 1989; Nairne, 1990), this is not the case for the mixed-list paradox. For example, although the Burgess and Hitch model predicts that mixing familiar and unfamiliar items in the same list will produce changes in patterns of performance relative to those for pure lists, the model continues to predict better recall of familiar items as opposed to unfamiliar items in mixed lists.

In studies of free recall, usually associated with longterm memory, it has been suggested that the mixed-list paradox may simply be a product of participant-controlled strategies whereby participants pay more attention to the encoding and rehearsal of low-frequency words because they believe that low-frequency words will be more difficult to remember. To test this view, M. J. Watkins, LeCompte, and Kim (2000) compared the effects of word frequency on free recall after either an incidental or an explicit learning task. They found that there was no reliable difference in the recall of high- and low-frequency words in mixed lists when an explicit learning procedure was used. However, high-frequency words were recalled better in both mixed and pure lists when an incidental learning 
task was used. They therefore suggested that the abolition of the word frequency effect in free recall of mixed lists depends on subjects' devoting differential attention to encoding low-frequency words when they are expecting a memory test. If such a strategy explanation could also explain the mixed-list paradox in serial recall (the abolition of the word frequency effect in mixed lists of high- and low-frequency words) this would greatly limit the theoretical importance of the effect.

The present study had two main goals. The first was to see whether this strategy-based hypothesis could account for the mixed-list paradox in serial recall. We used an incidental learning procedure that should eliminate the use of participant-controlled strategic processing. This led to the prediction that the usual high-frequency advantage found in pure lists should also occur in mixed lists under conditions of incidental learning. Conversely, in an intentional paradigm, the frequency effect should be apparent with pure lists and eliminated with mixed-frequency lists, because participants would revert to strategies favoring the encoding of low-frequency words. If this pattern of results were to be obtained, it would significantly reduce the theoretical importance of the mixed-list paradox. In fact, it would be difficult to maintain that this phenomenon can inform models of the basic processes involved in short-term memory for serial order, except perhaps by reminding us that strategic processing can override basic information-processing limitations.

The second aim of the present work was to verify whether the pattern of results obtained in immediate serial recall would be different with the introduction of a retention interval. In tasks requiring order information, benchmark findings have been shown to disappear or even reverse when recall is delayed. For example, Nairne and Kelley (1999) showed that with a reconstruction of order task, the advantage for dissimilar words in an immediate task was reversed following a brief period of distraction. This type of reversal has also been replicated with a serial recall task (Fournet, Juphard, Monnier, \& Roulin, 2003). If the mixed-list paradox was found to be present in both immediate and delayed serial recall tasks, this would support the generality of the effect. Conversely, if the mixed-list paradox was found to be absent in delayed recall, any explanation of the effect would have to account for this finding.

\section{EXPERIMENT 1}

In Experiment 1, we compared the immediate serial recall of mixed-frequency lists under incidental and intentional procedures. The participant strategy hypothesis predicted that high-frequency words should be recalled better in the incidental condition, but that this effect should be eliminated in the intentional condition because participants could choose to devote more resources to remembering the low-frequency words.

\section{Method}

Participants. Four groups of 37 participated in this experiment (once adjusted for removed participants): two groups in the inciden- tal procedure and two in the intentional procedure $(N=148)$. They were unpaid volunteers taking part in undergraduate psychology classes.

Materials. Six high- and 6 low-frequency words were used (see the Appendix). They were matched on imageability and number of syllables. The 12 words were used to create two lists: a mixed list beginning with a high-frequency word (HLHLHL) and a mixed list beginning with a low-frequency word (LHLHLH).

Procedure. Participants were tested in groups, in one 20-min session. The stimuli were presented on a screen at the front of the classroom. For the incidental groups (one for the HLHLHL list and one for the LHLHLH list), four practice trials and one critical trial were presented. The participants were required to remember the color and position of a series of squares. These squares could occupy one of nine positions in a $3 \times 3$ matrix and were all of a different color. Colored squares appeared one at a time, at a rate of $1 / \mathrm{sec}$. Following the presentation of a series of four squares, a warning signal was shown for $1 \mathrm{sec}$ and a further colored square was presented. This was the probe. The participants' task was to indicate whether the square previously presented in this position was of the same color as the probe. For the first two practice trials, this was the only task. Then word rating was introduced as a distractor task between the presentation and recall of the colored squares. The participants were told that this was to increase the difficulty of the colored-square memory task. They had to rate the imageability of six words on a 7-point scale ( 1 for low imageability and 7 for high imageability). Each word was presented for $3 \mathrm{sec}$, after which the colored-square probe occurred. After one such practice trial, the participants were told that the experiment proper was to start. They then had an additional practice trial that was identical to the one just described.

The following trial was the critical trial. Instead of being presented with the colored-square probe, the participants were given the instruction to recall, in order, as many as possible of the six words that they had just rated. They did so in writing on the back of the response sheet that they had been using for the color-probe and word-rating tasks. Finally, the participants were asked if they had anticipated this memory test. Participants who did not answer this question or who answered "yes" were eliminated from the study. For the intentional groups, the procedure was similar to that for a standard serial recall task: A list of words was presented, followed immediately by recall. The presentation rate was the same as in the incidental procedure.

\section{Results and Discussion}

Table 1 shows item and order recall means for each condition. A 2 (task) $\times 2$ (word frequency) ANOVA revealed an advantage for the intentional task [items in position, $F(1,146)=23.39, p<.001$; items irrespective of position, $F(1,146)=11.64, p<.001]$ but no significant effect of word frequency and no interaction between task and word frequency $\left(F_{\mathrm{S}}<1\right)$. The proportions of order errors per item recalled were also analyzed. This measure was computed by dividing the number of order errors by the total number of items recalled regardless of order (Murdock, 1976; Saint-Aubin \& Poirier, 1999). This measure revealed a significant advantage for the intentional task $[F(1,146)=10.33, p<.005]$, but no significant effect of word frequency and no significant interaction $[F<1$ and $F(1,146)=2.68, p=.10$, respectively].

In summary, none of the analyses showed a word frequency effect or an interaction between frequency and task, but the intentional task produced better item and order recall than did the incidental task. These results provide no support for the participant strategy hypothesis. 
Table 1

Mean Probability of Strict Serial Recall, Item Recall, Order Errors, and Proportion of Order Errors As a Function of Condition for Experiments 1, 2, and 3

\begin{tabular}{|c|c|c|c|c|c|c|c|c|}
\hline \multirow[b]{2}{*}{ Condition } & \multicolumn{2}{|c|}{ Strict Recall } & \multicolumn{2}{|c|}{ Item Recall } & \multicolumn{2}{|c|}{ Order Errors } & \multicolumn{2}{|c|}{ Prop. of Errors } \\
\hline & $M$ & $S D$ & $M$ & $S D$ & $M$ & $S D$ & $M$ & $S D$ \\
\hline \multicolumn{9}{|c|}{ Experiment $1:$ Incidental } \\
\hline Mixed HF & 0.31 & 0.26 & 0.55 & 0.27 & 1.46 & 1.45 & 0.41 & 0.41 \\
\hline Mixed LF & 0.33 & 0.28 & 0.53 & 0.28 & 1.22 & 1.44 & 0.34 & 0.41 \\
\hline \multicolumn{9}{|c|}{ Experiment 1 : Intentional } \\
\hline Mixed HF & 0.54 & 0.34 & 0.65 & 0.28 & 0.70 & 1.26 & 0.19 & 0.34 \\
\hline Mixed LF & 0.55 & 0.34 & 0.69 & 0.30 & 0.90 & 1.34 & 0.23 & 0.35 \\
\hline \multicolumn{9}{|l|}{ Experiment 2} \\
\hline Pure HF & 0.52 & 0.29 & 0.73 & 0.18 & 1.30 & 1.30 & 0.30 & 0.33 \\
\hline Pure LF & 0.40 & 0.23 & 0.58 & 0.24 & 1.13 & 0.93 & 0.35 & 0.30 \\
\hline Mixed HF & 0.39 & 0.31 & 0.59 & 0.27 & 1.18 & 1.64 & 0.32 & 0.41 \\
\hline Mixed LF & 0.40 & 0.31 & 0.61 & 0.29 & 1.21 & 1.46 & 0.31 & 0.37 \\
\hline \multicolumn{9}{|l|}{ Experiment 3} \\
\hline Pure HF & 0.51 & 0.29 & 0.70 & 0.21 & 1.12 & 1.37 & 0.28 & 0.35 \\
\hline Pure LF & 0.35 & 0.18 & 0.59 & 0.15 & 1.47 & 1.03 & 0.42 & 0.28 \\
\hline Mixed HF & 0.42 & 0.30 & 0.62 & 0.29 & 1.21 & 1.54 & 0.29 & 0.30 \\
\hline Mixed LF & 0.45 & 0.32 & 0.65 & 0.30 & 1.23 & 1.48 & 0.30 & 0.37 \\
\hline
\end{tabular}

Note-Strict recall refers to item in position, whereas item recall refers to item recall irrespective of order. HF, high frequency; LF, low frequency.

However, there is always the possibility that an incidental learning task does not produce a standard word frequency effect in immediate serial recall (i.e., an effect with pure lists).

\section{EXPERIMENT 2}

Experiment 2 was essentially a replication of the incidental conditions of Experiment 1, with the addition of pure high- and low-frequency lists to make sure that a wordfrequency effect could be obtained with pure lists. Participants recalled high-, low-, or mixed-frequency lists.

\section{Method}

Participants. Four groups of 38 participants took part in this study (once adjusted for removed participants). They were unpaid volunteers taking part in undergraduate classes.

Materials. New word lists (in French) were used. Six highfrequency and 6 low-frequency words were selected (see the Appendix). These 12 words were used to create four lists: a pure highfrequency list, a pure low-frequency list, a mixed list beginning with a high-frequency word (HLHLHL) and a mixed list beginning with a low-frequency word (LHLHLH). For the mixed-list conditions, the order of words in the pure lists was maintained, but the high- and low-frequency words were alternated. For example, the 1st, 3rd, and 5 th words in the HLHLHL list were the same words presented in the $1 \mathrm{st}, 3 \mathrm{rd}$, and 5 th positions of the pure high-frequency list.

Procedure. Three practice trials and one critical trial were presented. Each trial began with a series of nine colored squares presented one at a time (1,000 msec present, 1,000 msec absent). Six words were then presented on the screen, one at a time $(2,500 \mathrm{msec}$ present, $500 \mathrm{msec}$ absent) as distractors. The participants' task was to judge, on a 5-point scale, whether each word was pleasant (5) or unpleasant (1). Finally, 10 colored squares were presented sequentially (2,500 msec present, $500 \mathrm{msec}$ absent) for a yes/no recognition judgment. Participants were to answer "yes" when both the location and the color of a test square were recognized. On the fourth and critical trial, however, instead of making the series of recognition judgments, the participants were asked to recall the six words pre- sented as distractors in their order of appearance. Finally, the participants were asked whether they had anticipated the memory test. Those who gave no answer (4 participants) or who answered "yes" (3 participants) were eliminated from the study.

\section{Results and Discussion}

Table 1 presents the relevant means for Experiment 2. Because word frequency varied between subjects in the case of pure lists and within subjects in the case of mixed lists, the data were subjected to Erlebacher's (1977) ANOVA procedure. There was no significant effect of list type [items in position, $F(1,132)=1.77, p=.19$; items irrespective of position, $F(1,137)=2.94, p=.09$ ] or word frequency [items in position, $F(1,137)=3.12, p=$ .08 ; items irrespective of position, $F(1,141)=3.68, p=$ $.06]$, but there was a significant interaction between list type and word frequency for both measures $[F(1,137)=$ $4.72, p<.05$ for items in position, and $F(1,141)=5.90$, $p<.05$ for items irrespective of position]. High-frequency words were recalled better in pure lists for both measures $[t(74)=2.13, p<.05$, and $t(74)=3.06, p<.005$, respectively], but there was no difference between high- and low-frequency words in mixed lists $(t \mathrm{~s}<1)$. Finally, we considered the proportions of order errors. This analysis revealed that none of the main effects or the interaction was significant [word frequency, $F(1,149)=1.72, p=$ .19 ; list type and interaction, $F \mathrm{~s}<1]$.

These results established that with pure lists, a highfrequency advantage is observed with a serial recall procedure following incidental learning. Moreover, the results of Experiment 1 were replicated in that the highfrequency advantage was eliminated with the use of mixed lists. Hence, the mixed-list paradox for immediate serial recall was reliably reproduced under incidental learning; and the predictions of the participant strategy hypothesis were not supported. 


\section{EXPERIMENT 3}

As mentioned in the introduction, one of the aims of the present work was to verify whether the word frequency effect and the mixed-list paradox would generalize to a delayed serial recall task - or whether, as with other variables, a reversal of the pattern would be observed. To our knowledge, the effect of word frequency in delayed serial recall has never been assessed. Hence, Experiment 3 was a replication of Experiment 2 with the introduction of a delay between presentation and recall.

\begin{abstract}
Method
Participants. Four groups of 43 participants (once adjusted for removed participants) took part in this study $(N=172)$. They were unpaid volunteers taking part in undergraduate classes.

Materials and Procedure. The materials and procedure were identical to those of Experiment 2, with the sole exception that the yes/no recognition judgments were included on the critical trial in Experiment 3. These judgments served as a $30-\mathrm{sec}$ period of distraction between word ratings and word recall. Participants who indicated that they anticipated the memory test were eliminated $(n=4)$, as were those who did not answer $(n=12)$.
\end{abstract}

\section{Results and Discussion}

The results of Experiment 3 are shown in Table 1. Again, because word frequency varied between participants in the case of pure lists and within participants in the case of mixed lists, the data were subjected to Erlebacher's (1977) ANOVA procedure. For items in position, there was a significant main effect of word frequency $[F(1,167)=$ $4.01, p<.05]$, and there was no significant effect of list type $(F<1)$; but there was a significant interaction $[F(1,167)=8.52, p<.01]$, reflecting a clear advantage for high-frequency words in pure $[t(84)=3.19, p<.01]$, but not in mixed $(t<1)$, lists. An identical pattern was found for items irrespective of position with a significant effect of word frequency $[F(1,135)=11.75, p<.01]$ and no significant effect of list type $(F<1)$, but a significant interaction $[F(1,135)=4.46, p<.05]$. Once again, there was a clear effect of word frequency in pure lists $[t(84)=$ $2.78, p<.01]$, but not in mixed lists $(t<1)$. Finally, we considered the proportions of order errors. This revealed a significant effect of word frequency $[F(1,163)=3.78$, $p<.05]$, but the effect of list type $(F<1)$ and the interaction $[F(1,163)=2.70, p=.10]$ were not significant. Participants made more order errors per word recalled in the low-frequency condition.

The main objective of Experiment 3 was to verify whether the mixed-list paradox would generalize to a delayed serial recall task. The results showed that, contrary to other benchmark findings, the mixed-list paradox (and hence the word frequency effect) was not influenced by a retention interval.

\section{GENERAL DISCUSSION}

The three experiments reported here show that when high- and low-frequency items are mixed in the same list, the usual recall advantage for high-frequency words is eliminated. This pattern occurs when either incidental or intentional learning procedures are used, as well as in both immediate and delayed serial recall. These findings have important implications for models of serial recall, because explanations of the mixed-list effects in terms of participant-controlled strategies would lead us to expect the effects to disappear when incidental learning procedures are used.

Before we discuss the theoretical implications of the present data, two potential limitations of the studies must be addressed. The first concerns the presentation rate used here, which was slower than the one typically employed in serial recall experiments ( 1 item $/ 3 \mathrm{sec}$ instead of $1 \mathrm{item} / \mathrm{sec}$ ). One could ask whether our findings would generalize to faster, more typical presentation rates. Because participants' strategies do not appear to be a factor with a slow rate of presentation - where presumably they would be easier to deploy, given that there is more time available - there is no reason to expect that the pattern obtained here would not apply with faster rates of presentation as well.

Another issue concerns the apparent inconsistency of findings with free recall and serial recall. In a free recall task, M. J. Watkins et al. (2000) showed that the usual word frequency advantage was obtained in both pure and mixed lists in an incidental learning procedure, but that the frequency effect was abolished in mixed lists under explicit learning conditions. They interpreted this as support of participants devoting more effort to encoding the lowfrequency words in mixed lists when they were expecting a memory test. However, Dewhurst, Brandt, and Sharp (2004) obtained a different pattern of results using a similar procedure. Their study showed that the word frequency effect in free recall of mixed lists was abolished under incidental learning conditions. These results are more in line with those obtained in the present experiments and do not lend support to the encoding strategy hypothesis in the case of free recall. Although this is beyond the scope of the present paper, future research should revisit the mixedlist paradox in free recall in an attempt to resolve current inconsistencies.

If the mixed-list paradox in immediate serial recall is not attributable to encoding strategies, how shall we account for it? The dominant interpretation of the standard high-frequency advantage has been that it is the result of redintegration at retrieval (e.g., Saint-Aubin \& Poirier, 2000; Schweickert, 1993). Most forms of this hypothesis suggest that the phonological representations underlying list recall are subject to degradation. At the point of recall, items must be redintegrated or "cleaned-up"- a process thought to call on long-term knowledge of the language. The probability of successful redintegration and recall depends on the ease with which that item's long-term representation can be accessed. High-frequency items, in this view, are recalled better because their representations in long-term memory are more easily retrieved. An itembased redintegration hypothesis cannot readily account 
for the absence of a word frequency effect in mixed lists without making the additional assumption that redintegration of any given item must depend on the list context in which the item occurs.

Hulme et al. (2003) have described a new version of the redintegration hypothesis developed to account for mixed-list findings within a serial recall task. According to the co-occurrence hypothesis, the mixed-list paradox would reflect a contribution from preexisting interitem associations between the lexical/semantic representations of items within a list. Because high-frequency items tend to co-occur more often in the language, pure highfrequency lists benefit from a coactivation process: Items that co-occur in the language will tend to heighten each other's accessibility at the point of retrieval. In mixed lists as opposed to pure lists, interitem associations between high-frequency items would be reduced, whereas the lowfrequency items would benefit; the probability of a lowfrequency item co-occurring with a high-frequency item is higher than the probability of a low-frequency item cooccurring with another low-frequency item.

Finally, another proposal could account for the results presented here. The item-order hypothesis (Nairne, Riegler, \& Serra, 1991; Serra \& Nairne, 1993), developed to account for free recall findings, assumes that there are limited resources available to encode two important types of information-namely, item-specific information and relational information. This proposal suggests that the mixed-list paradox is produced by a trade-off between item and order information processing. A further assumption is that recall is guided by relational or order information. Basically, the suggestion of the item-order hypothesis is that low-frequency items automatically involve more resources being attributed to item-specific encoding, leaving fewer resources for order information encoding. If one accepts that order information is important in that it guides recall, then this item-order hypothesis can account for the usual high-frequency advantage: Order information is encoded better for high-frequency items, and this overshadows the item encoding advantage of low-frequency items because order is important in retrieval. In the case of mixed lists, the item-order hypothesis suggests that the combination of high- and low-frequency items evens out order information encoding, making it less efficient for high-frequency items and more efficient for low-frequency items. This allows the better item encoding of the low-frequency items to affect performance to a greater degree. Hence, the prediction is that in comparison with pure lists, mixed lists will involve a significant reduction or even a reversal of the high-frequency advantage.

However, to properly assess such a proposal, item information would need to be measured with a task not requiring order information (e.g., recognition), whereas order information should be measured with a task placing heavy demands on order information (e.g., serial recall, order reconstruction). In our experiments, however, both of our measures involved order information. If the correct in position scoring is used as an order measure, the predic- tions of the item-order hypothesis are supported. In effect, in our experiments, high-frequency words were recalled better than low-frequency words in pure lists, whereas no difference was found in mixed lists.

Both of these proposals can account for the findings reported here, and choosing between them will require further research. In particular, to test the item-order hypothesis it will be necessary to compare directly measures of order memory (e.g., serial recall) with measures of memory that do not require order information (e.g., item recognition) using mixed and pure lists.

\section{Conclusion}

We have reported three experiments examining the predictions of the participant strategy hypothesis for the word frequency effect in intentional and incidental serial memory performance. The results allow us to discard the suggestion that participant-controlled strategies are responsible for the mixed-list paradox observed in immediate serial recall when item frequency is manipulated. Moreover, our results indicate that this is also true when a delay is introduced, implying that whatever mechanism is involved may be a relatively general one, perhaps involved in short-term as well as longer term serial memory. We have briefly discussed two promising accounts - namely, the item-order hypothesis and the co-occurrence hypothesis. We would suggest that further work examining mixed lists and other contextual effects in immediate and delayed serial recall should allow us to determine the better explanation of the mixed-list paradox.

\section{REFERENCES}

Burgess, N., \& Hitch, G. J. (1999). Memory for serial order: A network model of the phonological loop and its timing. Psychological Review, 106, 551-581.

Dewhurst, S. A., Brandt, K. R., \& Sharp, M. S. (2004). Intention to learn influences the word frequency effect in recall but not in recognition memory. Memory \& Cognition, 32, 1316-1325.

ERLEBACHER, A. (1977). Design and analysis of experiments contrasting the within- and between-subjects manipulation of the independent variable. Psychological Bulletin, 84, 212-219.

Fournet, N., Juphard, A., Monnier, C., \& Roulin, J.-L. (2003). Phonological similarity in free and serial recall: The effect of increasing retention intervals. International Journal of Psychology, 38, 384-389.

Gregg, V. H., Freedman, C. M., \& Smith, D. K. (1989). Word frequency, articulatory suppression and memory span. British Journal of Psychology, 80, 363-374.

Hulme, C., Stuart, G., Brown, G. D. A., \& Morin, C. (2003). Highand low-frequency words are recalled equally well in alternating lists: Evidence for associative effects in serial recall. Journal of Memory \& Language, 49, 500-518.

LeWANdowsky, S., \& Murdock, B. B., JR. (1989). Memory for serial order. Psychological Review, 96, 25-58.

Murdock, B. B., JR. (1976). Item and order information in short-term serial memory. Journal of Experimental Psychology: General, 105, 191216.

NAIRne, J. S. (1990). A feature model of immediate memory. Memory \& Cognition, 18, 251-269.

Nairne, J. S., \& Kelley, M. R. (1999). Reversing the phonological similarity effect. Memory \& Cognition, 27, 45-53.

Nairne, J. S., Riegler, G. L., \& Serra, M. (1991). Dissociative effects of generation on item and order retention. Journal of Experimental Psychology: Learning, Memory, \& Cognition, 17, 702-709. 
Poirier, M., \& SAINT-Aubin, J. (1996). Immediate serial recall, word frequency, item identity and item position. Canadian Journal of Experimental Psychology, 50, 408-412.

Saint-Aubin, J., \& Poirier, M. (1999). The influence of long-term memory factors on immediate serial recall: An item and order analysis. International Journal of Psychology, 34, 347-352.

SAINT-Aubin, J., \& PoIRIER, M. (2000). Immediate serial recall of words and nonwords: Tests of the retrieval-based hypothesis. Psychonomic Bulletin \& Review, 7, 332-340.

SCHWEICKERT, R. (1993). A multinomial processing tree model for degradation and redintegration in immediate recall. Memory \& Cognition, 21, 168-175.

Serra, M., \& NaIrne, J. S. (1993). Design controversies and the genera- tion effect: Support for an item-order hypothesis. Memory \& Cognition, 21, 34-40.

Stuart, G., \& Hulme, C. (2000). The effects of word co-occurrence on short-term memory: Associative links in long-term memory affect short-term memory performance. Journal of Experimental Psychology: Learning, Memory, \& Cognition, 26, 796-802.

Watkins, M. J., LeCompte, D. C., \& Kim, K. (2000). Role of study strategy in recall of mixed lists of common and rare words. Journal of Experimental Psychology: Learning, Memory, \& Cognition, 26, 239-245.

WATKINS, O. C., \& WATKINS, M. J. (1977). Serial recall and the modality effect: Effects of word frequency. Journal of Experimental Psychology: Human Learning \& Memory, 3, 712-718.

\section{APPENDIX A}

Stimuli Used in Experiments 1-3

\begin{tabular}{|c|c|c|c|c|}
\hline \multicolumn{5}{|c|}{ Experiment 1} \\
\hline $\begin{array}{l}\text { High-Frequency Words } \\
\text { family }\end{array}$ & telephone & government & decision & experience \\
\hline $\begin{array}{l}\text { Low-Frequency Words } \\
\text { emperor pioneer }\end{array}$ & bayonet & subaltern & pyramid & disparity \\
\hline \multicolumn{5}{|c|}{ Experiments 2 and 3} \\
\hline $\begin{array}{ll}\text { High-Frequency Words } \\
\text { semaine } & \text { journée } \\
\text { [week }] & {[\text { day }]}\end{array}$ & $\begin{array}{l}\text { argent } \\
{[\text { money }]}\end{array}$ & $\begin{array}{l}\text { problème } \\
\text { [problem] }\end{array}$ & $\begin{array}{l}\text { musique } \\
\text { [music] }\end{array}$ & $\begin{array}{l}\text { famille } \\
\text { [family] }\end{array}$ \\
\hline $\begin{array}{ll}\text { Low-Frequency Words } & \\
\text { moquette } & \text { refuge } \\
\text { [carpet] } & \text { [refuge }]\end{array}$ & $\begin{array}{l}\text { sifflet } \\
\text { [whistle] }\end{array}$ & $\begin{array}{l}\text { angoisse } \\
\text { [anguish] }\end{array}$ & $\begin{array}{l}\text { cannelle } \\
\text { [cinnamon] }\end{array}$ & $\begin{array}{l}\text { lutin } \\
\text { [imp] }\end{array}$ \\
\hline
\end{tabular}

(Manuscript received April 1, 2004;

revision accepted for publication December 12, 2005.) 